\title{
Characterisation and investigation of local variations in mechanical behaviour in cast aluminium using gradient solidification, digital image correlation and finite element simulation
}

\author{
Jakob Olofsson ${ }^{\mathrm{a},},{ }^{*}$, Ingvar L. Svensson ${ }^{\mathrm{a}}$, Pascal Lava $^{\mathrm{b}}$, Dimitri Debruyne $^{\mathrm{b}}$ \\ ${ }^{a}$ Materials and Manufacturing - Casting, Department of Mechanical Engineering, School of \\ Engineering, Jönköping University. P.O. Box 1026, SE-551 11 Jönköping, Sweden \\ ${ }^{\mathrm{b}}$ Department MTM, KU Leuven, Kasteelpark Arenberg 44, B-3001 Leuven, Belgium.
}

* Corresponding author. E-mail address: jakob.olofsson@jth.hj.se Tel: +46 36101659.

\begin{abstract}
Due to design and process-related factors, there are local variations in the microstructure and mechanical behaviour of cast components. This work establishes a Digital Image Correlation (DIC) based method for characterisation and investigation of the effects of such local variations on the behaviour of a high pressure, die cast (HPDC) aluminium alloy. Plastic behaviour is studied using gradient solidified samples and characterisation models for the parameters of the Hollomon equation are developed, based on microstructural refinement. Samples with controlled microstructural variations are produced and the observed DIC strain field is compared with Finite Element Method (FEM) simulation results. The results show that the DIC based method can be applied to characterise local mechanical behaviour with high accuracy. The microstructural variations are observed to cause a redistribution of strain during tensile loading. This redistribution of strain can be predicted in the FEM simulation by incorporating local mechanical behaviour using the developed characterization model. A homogeneous FEM simulation is unable to predict the observed behaviour. The results motivate the application of a previously proposed simulation strategy, which is able to predict and incorporate local variations in mechanical behaviour into FEM simulations already in the design process for cast components.
\end{abstract}

\section{Keywords}

Casting; Component behaviour; Mechanical behaviour; Aluminium; Digital Image Correlation; Plastic behaviour.

\section{Introduction}

Casting is a powerful manufacturing method that enables the design and manufacturing of products and components with several desirable properties, e.g. complex geometries, near net shape and cost efficiency. Although casting has been known and used for thousands of years, the last few years has seen it evolve into a highly advanced and modern production method which is highly utilised in e.g. the automotive and transportation sectors [1]. As demands for reduced emission levels and fuel 
consumption increases, it becomes important to achieve full utilisation of all features of the casting process in order to enable design and production of optimised and robust components.

The solidification of cast components involves several complex phenomena, where factors such as component geometry, chemical composition, heat transfer and solidification kinetics affect the extraction of heat during solidification. This leads to local variations in solidification conditions, microstructure and material behaviour of the solidified casting and results in cast components which display local variations in mechanical behaviour rather than homogeneous material behaviour [2, 3]. In cast aluminium components, local mechanical behaviour is highly related to local cooling rates during solidification. A high local cooling rate yields a highly refined microstructure, typically measured by determining Secondary Dendrite Arm Spacing (SDAS). A high cooling rate yields a low SDAS-value while a lower cooling rate yields a higher SDAS-value [4, 5]. High microstructural refinement is generally associated with high strength, whereas decreasing the SDAS-value causes a general increase in yield strength, ultimate tensile strength and ductility for as-cast materials [4-9]. However, there may be complex variations of these trends because the cooling rate also affects the solidification conditions of other microstructural features $[9,10]$. For example, an increased cooling rate refines the eutectic silicon particles [11], strengthens the dendrites due to silicon enrichment [12] and affects the segregation profiles within the different phases [13].

In recent years, extensive research has been conducted in the field of modelling and simulation of solidification in cast aluminium components. Solidification models have been developed which calculates the phase diagram, cooling curves and solidification behaviour to predict microstructural features locally throughout the component based on the chemical composition of the alloy [14]. By implementing these models into casting simulation software, it is possible to determine the local variations in microstructural features throughout cast components with high accuracy $[3,14,15]$. By relating microstructure and mechanical behaviour using material characterisation models, local mechanical behaviour can be predicted [3]. These simulation tools provide the basis for a simulation strategy denoted the closed chain of simulations for cast components which has recently been presented by two of the authors of this work [16]. The strategy, schematically illustrated in Fig. 1, connects and utilises numerical solidification models and casting process simulations to predict local variations in microstructure and mechanical behaviour and then incorporates this data in a Finite Element Method (FEM) simulation of the behaviour of the cast component [16]. The simulation strategy includes characterisation models developed for cast iron [17] and sand cast aluminium alloys $[2,3]$, whereas models for High Pressure Die Cast (HPDC) aluminium alloys have not been previously established.

A numerical investigation of a ductile iron component using the proposed simulation strategy has shown that the predicted behaviour of the component when subjected to load is significantly affected by local variations in mechanical behaviour, which cause a redistribution of stresses and strains that it is impossible for a model in a homogenous material to predict [18]. However, this effect has only been predicted numerically, not confirmed experimentally.

The mechanical behaviour of a cast alloy is typically characterised by a tensile curve obtained through a tensile test, where the obtained strain is plotted versus applied load. The strain is typically measured using a contact extensometer or a non-contact laser extensometer. However, both these methods measure the average strain over a gauge area and are unable to display the complex 
variation in the strain field of the specimen [19]. Digital Image Correlation (DIC) is an optical noncontact deformation measurement method that offers the opportunity to study the full displacement and strain field for a specimen. Usually, a speckled pattern is applied to the specimen and by observing the displacement of the pattern when a load is applied, it is possible to calculate the displayed strain field in the specimen using a DIC algorithm. The method can be applied on different scale levels, e.g. on the macroscopic level (entire samples/components) or on the microscopic level (using the natural pattern in micrographs). The accuracy and precision of DIC in elasto-plasticity has been the subject of intensive research and development in recent years [20-22] and has been used in various applications for material characterisation, e.g. inverse methodologies [19, 23], internal and external work comparison [24] or virtual fields methodologies [25]. Regarding cast materials, DIC has e.g. been applied on a microstructural level to study the distribution of strain over different phases during tensile loading of cast irons [26] and on a macroscopic level to study the fracture of a HPDC aluminium alloy [27]. However, the use of DIC to characterise or investigate the macroscopic effects of local variations in microstructure caused by local variations in solidification conditions is to the authors' knowledge not reported in the literature.

In this work, the effects of local variations in microstructure and mechanical behaviour in a HPDC aluminium alloy is characterised and investigated. Two sets of gradient solidified specimens were produced. The first set was produced to obtain specimens of high microstructural homogeneity. This set was used for characterisation of mechanical behaviour at three different solidification conditions. The second set was produced to display a controlled and repeatable variation in microstructure throughout the specimens and was used to study the effects of local variations in microstructure on the strain field observed using DIC. This contribution aims to extend the characterisation models available in the closed chain of simulations for cast components to include HPDC alloys and also to experimentally investigate the numerically predicted redistribution of strain. Local variations in mechanical behaviour directly affect both the performance and optimal design of cast components; incorporating these variations into the design process of cast components will improve the design process of optimised and robust cast components. Thus, the results of the current work directly affect not only the work of design and CAE engineers, but also that of metallurgists and foundry engineers.

\section{Experimental setup}

\section{I. Material}

The alloy under investigation is a typical alloy used for HPDC components and meets the EN AC 46000 alloy standard. It contains a relatively high iron (Fe) content, 0.6-1.1\%, and although this reduces the problem of die soldering in permanent moulds, there is a risk that formation of iron-rich intermetallics will occur during solidification, which is detrimental to the mechanical properties of the material [28]. In the current work, the alloy has been studied using a gradient solidification method, in which cylindrical samples are remelted and solidified using a furnace mounted on an electric lifting device. Setting the velocity at which the furnace is lifted provides control over the cooling rate and, thus, the microstructural refinement obtained in the samples. Except for providing a controlled and repeatable process for producing materials with microstructures which are highly homogeneous, the gradient solidification method also has the advantage of producing materials with low content of oxide films, low porosity and low degree of shrinkage related defects. The layout of 
the equipment is further described elsewhere [29] and the technique has been extensively applied in investigations and characterisations of the behaviour of cast aluminium alloys [2, 6, 7, 12, 29]. As previously pointed out, it is important to stress that the value of SDAS is not the only parameter affected by changes in cooling rates. However, SDAS is an accepted measure of the microstructural refinement in as-cast aluminium.

The base material was taken from a component manufactured in serial production at a HPDC foundry. The material was remelted in an induction furnace and cast into cylindrical samples, each casting producing six samples. A sample for chemical analysis was also made and the chemical composition was measured using a Spectro Spectromax optical emission spectrometer. The average chemical composition obtained from three measurements is shown in Table 1 . The cylindrical samples were preheated in a holding furnace at $125^{\circ} \mathrm{C}$ for at least 30 minutes. Three samples were then placed in steel pipes and put into the gradient solidification furnace. The operating temperature of the gradient furnace was $715^{\circ} \mathrm{C}$. The samples were initially heated for 30 minutes in order to achieve total remelting of the material prior to lifting the furnace at a defined speed. This caused directional solidification of the cast sample from the bottom of the steel pipe upwards.

In this work, two sets of specimens were produced. The first set was produced using constant lifting speed of the furnace, which provided a constant temperature gradient in the sample holder during solidification. This leads to a microstructure with high microstructural homogeneity throughout the sample. The samples of the first set were used to determine the relationship between the coarseness of the microstructure and the mechanical behaviour of the material. Three experiment series with different cooling rates were produced: $3 \mathrm{~mm} / \mathrm{s}, 0.3 \mathrm{~mm} / \mathrm{s}$ and $0.03 \mathrm{~mm} / \mathrm{s}$, which generally corresponds to SDAS-values of about $10 \mu \mathrm{m}, 25 \mu \mathrm{m}$ and $50 \mu \mathrm{m}$, respectively. These three series are henceforth referred to as SDAS10, SDAS25 and SDAS50. Six samples per cooling rate were produced, giving a total of eighteen samples. It should be pointed out that while fine and intermediate microstructures can typically be found in HPDC components, coarse microstructures are rather representative of the microstructure of sand cast components [3]. For the purposes of material characterisation, however, it is of interest to study the behaviour of the alloy for this condition as well.

The second experimental set was produced by changing the speed of the furnace during solidification, causing a variation in the local cooling rate during solidification. Thus, a variation in the microstructure throughout the sample was obtained. For this set of samples, the lifting speed of the furnace accelerated from a low initial velocity for the lower part of the specimen to a higher speed for its upper region, resulting in a transition from an intermediate microstructural refinement to a fine microstructure within the specimen. A series containing six samples was produced, henceforth referred to as the SDASVAR series.

Samples intended for evaluation of microstructure were prepared and studied using optical microscopy. SDAS is not always easily measured or accurately determined. In this work, focus was placed on secondary dendrites likely to have grown from the same primary dendrite arm. The length of a line was measured from the centre of one secondary dendrite to another, passing over at least one intermediate secondary dendrite arm and perpendicular to the growth direction of the measured secondary dendrites. The SDAS value was then estimated by dividing the length of the line with the number of dendrite arm spacings covered by the line. One sample per series was measured 
at five different heights of the tensile specimens. The reported values are average values from at least three measurements for each position.

\subsection{Tensile testing}

Flat tensile specimens were prepared according to the standards of ASTM: B557M-10 with a specimen thickness of $4 \mathrm{~mm}$, a width of $6 \mathrm{~mm}$ and a reduced section length of $32 \mathrm{~mm}$. Tensile testing was performed using a Zwick/Roell Z100 testing machine with a $100 \mathrm{kN}$ load cell at room temperature, using a crosshead speed of $0.35 \mathrm{~mm} / \mathrm{min}$. Strain measurements were made using a Zwick/Roell laser extensometer with an initial distance of $26 \mathrm{~mm}$ between the measuring points.

The obtained tensile curve is characterised using Hooke's law $\left(\sigma=E \cdot \varepsilon_{e l}\right)$ for the elastic regime. Here $\sigma$ is the true stress (Pa), E is the Young's modulus and $\varepsilon_{e l}(-)$ the elastic strain. For the plastic regime, there are many models available [30]. In the current work, the Hollomon equation [31] was used. The Hollomon equation relates the true stress and the true plastic strain $\varepsilon_{p l}$ as

$$
\sigma=K \times \varepsilon_{p l}^{n}
$$

where $n$ is the strain hardening exponent $(-)$ and $K(\mathrm{~Pa})$ is commonly referred to as the strength coefficient. The strain hardening exponent ranges from zero to one, where $n=0$ corresponds to a perfectly plastic material and $n=1$ corresponds to a linear deformation hardening material. The applicability of the Hollomon equation has been previously investigated and generally provides a good description of plastic behaviour for as-cast aluminium alloys [32, 33]. The parameters of the Hollomon equation can be determined using a double logarithmic plot of Eq. (1). If the plastic behaviour of the material follows the Hollomon equation, such a plot yields a straight line where $n$ is the slope of the line and $K$ is the stress level where the true plastic strain equals unity. In this work, this evaluation was performed using MATLAB [34].

\subsection{Digital Image Correlation setup}

The images for the DIC-measurements were captured by a camera mounted on the tensile testing machine. The camera was positioned so that it could observe one of the flat surfaces of the specimen while the laser extensometer was irradiating the other. The camera is mounted perpendicular to the specimen and the deformation of the flat specimen is in plane, which enables the use of 2D DIC. The setup is schematically illustrated in Fig. 2. A pattern was applied to the surface of the specimen using spray paint, starting with a white bottom layer followed by a black, speckled pattern. Images with a resolution of $1624 * 1280$ pixels were captured at a frame rate of $10 \mathrm{~Hz}$ using an AVT Stingray F201B 8-bit camera with a Tamron M118FM25 lens (focal length $25 \mathrm{~mm}$ ). Synchronization with the tensile device was performed manually due to limitations with the experimental equipment. The MatchID Grabber [35] software was used for frame grabbing, and the MatchID 2D [35] software was used for correlation calculations using the general parameters shown in Table 2. Evaluation of the results was performed using MatchID [35] 2D and MATLAB [34].

A systematic method was applied in which the strains measured by DIC were used to characterise the material behaviour and also to study the variations in the obtained strain field. Although the DIC software is able to provide a full-field analysis of the strain field for the entire specimen, only the central part of the reduced section of the specimen, where a uniaxial stress field is obtained, was 
used for characterising the mechanical behaviour of the material subjected to uniaxial load. Thus, the average value for the strain distributed over this section of the tensile specimen was used as the strain measurement for material characterisation, referred to as the MAIN measurement. This area corresponds to the area measured by the laser extensometer on the opposite side of the sample. In order to facilitate the study of variations in the strain field in this area, it was divided into three gauge areas: TOP, MIDDLE and BOTTOM, see Fig. 3. The width and height of the square gauge regions are about $6 \mathrm{~mm}$, which is larger than the spatial strain resolution in the DIC correlations (about $2.8 \mathrm{~mm}$ ). The amount of variation in the strain field is evaluated using an average relative strain variation, ASV (\%), calculated as

$$
A S V=\frac{100}{m} \sum_{m} \frac{\varepsilon_{\max }-\varepsilon_{\min }}{\varepsilon_{\text {MAIN }}}
$$

where $\varepsilon_{\max }$ and $\varepsilon_{\min }$ are at every sampling point the maximum and minimum values of the average strain values for the TOP, MIDDLE or BOTTOM areas, $\varepsilon_{\text {MAIN }}$ is the average strain value for the MAIN area, and the average value over the $m$ number of sampling points is calculated. Thus, a high ASV value indicates a large variation in the measured strain field during the tensile test.

\subsection{FEM simulation}

A FEM model of the tensile specimens was created in order to verify the applicability of the modelling of the local variations in mechanical behaviour. In order to be able to implement various material definitions throughout the specimen and simulate the effects of local variations in mechanical behaviour, a three-dimensional model of the entire specimen was created with no assumptions regarding symmetry in the boundary conditions. The mesh consisted of about 3000 hexagonal second order elements (C3D20R in Abaqus [36]). Boundary conditions were applied at the upper and lower parts of the specimen. A linearly increasing displacement was applied to simulate the behaviour of physical tensile testing, and the von Mises yield criterion was used. The simulations were performed using the Abaqus [36] implicit solver.

Material definitions for the FEM model were created using previously developed, in-house software [16]. The software creates element individual material properties, i.e. one material definition for every element of the FEM mesh. This software has previously been applied to create element individual material definitions for cast components based on casting process simulation results [16, 18 , 37]. In this work, characterisation models relating the coarseness of the microstructure and the local mechanical behaviour were established using the tensile testing results. The microstructural refinement, as measured by optical microscopy on different heights of the tensile specimens, was then used as input for the characterisation models to predict the elemental value of the parameters of the Hollomon equation. A layered approach was used in which the SDAS measurements served as input for the elements for each of the measuring heights and the SDAS-value was linearly interpolated for the intermediate layers. This way, a FEM model containing local variations in mechanical behaviour was obtained, based on the physically observed microstructural variations.

\section{Results and discussion}

\section{I. Microstructure}


The obtained microstructure in the MIDDLE area of samples from the three constant cooling rate series is shown in Fig. 4. As has been previously discussed, it is clear that not only SDAS but also other microstructural features, such as the morphology of the eutectic Si precipitates and iron rich intermetallics, were altered by changes in cooling rate. The SDAS measurements verify that SDAS of about 10, 25 and $50 \mu \mathrm{m}$ were obtained. No clear variations in SDAS over the height of the specimens were noted, indicating a high level of homogeneity throughout the samples. This is consistent with previous studies using the gradient solidification equipment $[2,6,7,12,29]$.

The variations in cooling rate applied for the SDASVAR series caused clear variations in microstructure over the gauge areas of the specimen (Fig. 5), where the SDAS-values for the BOTTOM, MIDDLE and TOP areas were measured to 20,15 and $10 \mu \mathrm{m}$, respectively. This variation can be considered to be representative of local variations in microstructure found in actual HPDC components.

\subsection{Material characterisation}

The tensile curves obtained for the specimens with constant microstructural refinements are shown in Fig. 6. The different microstructural refinements display significant differences in mechanical behaviour and both ultimate tensile strength and ductility decrease significantly with an increasing SDAS-value (Fig. 7a). This is consistent with previous findings [2, 6, 7, 12, 29]. It was also noted that the intermediate microstructure has a somewhat higher hardening rate than the fine microstructure at low plastic strains, but lower at higher plastic strains. Thus, the $0.2 \%$ offset yield strength is found to be higher for the intermediate microstructural refinement of the SDAS25 series than for the fine microstructure of the SDAS10 series and lower for the SDAS50 series. This behaviour is explained by a solid solution hardening effect caused by an increased level of magnesium atoms in solid solution in the dendrites due to back diffusion. Using wavelength dispersive spectroscopy (WDS) measurements, this effect has previously been observed to be more pronounced for intermediate microstructural refinement [13] and is expected to affect deformation behaviour at low strain levels [38].

The evaluated values of the parameters of the Hollomon equation as a function of SDAS is shown in Fig. 8; Fig. 8a shows variations for the strain hardening exponent, $n$, and Fig. $8 \mathrm{~b}$ variations for the strength coefficient, $K$. There is a generally good agreement between the results in this work and the results reported for other cast aluminium alloys in the literature $[2,6,7,12,29]$.

To characterise the relationship between the parameters of the Hollomon equation and the SDASvalue, Seifeddine and Svensson [2] found that characterisation models on the forms

$$
\begin{aligned}
& n=A \times \ln (S D A S)+B \\
& K=C \times \ln (S D A S)+D
\end{aligned}
$$

provided a good fit to their experimental data. For alloys with different iron contents, parameters A$D$ where reported to be linearly dependent on the iron content [2]. In this work, only one level of iron content was studied, why the values of parameters A-D are constants evaluated from the measurements. An equation on the form in Eq. 3 is found to provide a very good fit to the gathered experimental data, with a coefficient of determination of $R^{2}=0.9997$. The model is represented by dashed lines in Fig. 8a. However, the results for the strength coefficient show an almost linearly decreasing dependency on the SDAS-value; a relationship on the form in Eq. 4 is unable to provide a 
fit with the current data. Instead, a relationship between the strength coefficient $K$ and the SDASvalue on the exponential form

$$
K=E \times \exp (F \times S D A S)
$$

has been found to provide a good fit with a coefficient of determination of $R^{2}=0.9977$. The constants $E$ and $F$ have been evaluated from the experimental data. A comparison between the fit of the two models (Eq. 4 and Eq. 5) is shown in Fig. 8b. In the following, a characterisation model based on Eq. 3 and Eq. 5 is applied.

A comparison between the tensile curve obtained using the laser extensometer and the DIC strain measurements in the MAIN area is shown in Fig. 9. There is a very good agreement between the two curves for all microstructures, indicating a high degree of conformity between the two measurement methods. The evaluated parameters of the Hollomon equation based on the DIC measurements are reported in Table 3. Both the laser and DIC measurements are found to give the exact same results for the strain hardening exponent and the values for the strength coefficient display only small numerical differences falling within measured standard deviations. These results show that the DIC measurements are highly accurate and can be applied to characterise the relationship between microstructure and mechanical behaviour for cast aluminium alloys.

The calculated value of the average strain variation from the DIC measurements, listed in , was found to be low for low and intermediate SDAS-values. However, for the coarse microstructure, SDAS50, the value is significantly higher, indicating a larger variation in the strain field. This is also qualitatively seen in the DIC strain images of the full strain field in the specimens (Fig. 10). The SDAS10 and SDAS25 series both display a rather even distribution of strain throughout the specimens, whereas a large local strain concentration is clearly seen already at low loads in the SDAS50 series. This alloy contains a high amount of iron, which during solidification can form iron-rich intermetallics, especially in microstructures formed at low cooling rates. These iron rich intermetallics are highly detrimental to the mechanical behaviour [28]. Visual inspection of the fracture surface also reveals that large particles were present in locations where strain concentrations were detected. Thus, it is not necessary for all variations in the observed strain field to be related to variations in the microstructural refinement; they may also be caused by the presence of features such as iron rich intermetallics and other defects.

It is noted in Fig. 10 that the SDAS25 series shows a slightly lower strain at this load compared to the SDAS10 series, which is consistent with the behaviour previously noted in Fig. 6 .

\subsection{Variations in microstructure and mechanical behaviour}

The previously described layered material definitions method has been applied to incorporate local variations in mechanical behaviour into the FEM model. Thus, the material behaviour in the FEM model is not homogenous; instead, the local variations in mechanical behaviour are implemented based on the measured values of SDAS at different heights of the tensile sample and the developed characterisation model (Eq. 3 and Eq. 5). This simulation is henceforth referred to as the LOCAL simulation. 
For comparison, a simulation has also been performed using a homogeneous material description based on the SDAS-value at the centre of the specimen (SDAS $15 \mu \mathrm{m}$ ). This simulation is henceforth referred to as the HOMOGENEOUS simulation.

Fig. 11 and Fig. 12 show comparisons between the strain fields for the SDASVAR series observed using DIC and predicted by the LOCAL and HOMOGENEOUS simulations for a low and a high plastic strain regime, respectively. The DIC strain field shows that the coarser microstructure in the bottom area exhibits a lower level of strain at a given load in the low plastic load strain regime (Fig. 11). This is consistent with the findings in Fig. 6, where it is seen that the tensile curve of the intermediate microstructure initially exhibits a lower level of strain for a given load than a fine microstructure does. As the load increases, however, the strain is redistributed and in the higher plastic load strain regime (Fig. 12), the intermediate microstructure instead exhibits a higher level of strain than the finer microstructure does. All samples of the SDASVAR series are seen to fail in the bottom region, where the coarser microstructure both exhibits the highest strain level (Fig. 12) and the lowest ductility (Fig. 7). Thus, variations in microstructure cause localisation and redistribution of strain within the sample during tensile testing.

As seen in Table 4, the average strain variation, ASV, is about three times higher for the SDASVAR series than for the other series in the same SDAS range, i.e. SDAS10 and SDAS25. This indicates that variations within the strain field of the sample are too large to be explained by statistical deviation and that it is actually related to the very variation in microstructure.

Small, recurring variations in the distribution of strain can be observed as horizontal bands across the DIC strain fields. To varying extent, these bands are visible for all samples at low plastic strains (Fig. 10 and Fig. 11). At higher plastic strains, the variations become less pronounced (Fig. 12). It is not clear whether this is due to aliasing in the DIC measurements or a real physical effect, but this will be further investigated in future work.

The redistribution of strain to different areas can be seen in the FEM simulation results obtained by the LOCAL simulation (see Fig. 11 and Fig. 12). There is a very good agreement between the LOCAL simulation and the DIC measurements regarding prediction of the amount of strain in the different areas of the specimen, both in the low and high plastic strain regimes, and the redistribution of strain at different loads is well described. The HOMOGENEOUS simulation, however, is unable to predict the strain redistribution phenomena. Since homogeneous material behaviour is assumed the predicted strain distribution will always be symmetrical, i.e. the upper and the lower parts of the specimen are predicted as the regions subjected to the highest strain.

Thus, the strain redistribution effect that has previously been numerically predicted by FEM simulations $[18,37]$ has been experimentally confirmed in the current work. To the authors' knowledge, no similar observations have been reported in the literature. The DIC based method is found to be a good tool to improve understanding of the effects of local variations in microstructure and will be further developed in future work.

The results show that incorporation of the local variations in mechanical behaviour significantly increases the predictive power of the FEM simulations to predict the correct strain distribution. These variations does not only occur in tensile specimens, but also to varying extents in cast components, e.g. in geometric transitions where variations may occur both in the microstructure and 
the stress field due to geometric stress concentrations. A homogeneous material description is unable to correctly predict the strain field and may cause incorrect conclusions to be drawn regarding the performance of the component. Although only static properties have been studied in this work, correct prediction of the strain field throughout the component is crucial also in simulations studying damage, crack propagation or fatigue. An incorrectly predicted strain field may e.g. cause incorrect selection of locations for the initiation of cracks or incorrect prediction of the lifetime of the components. Predicting variations in microstructure and incorporating them into the FEM simulations using the closed chain of simulations for cast components will facilitate a more realistic prediction of the actual stress and strain distribution within a component in use. Determination of the optimal component geometry of the component, in which the effect of the casting process and solidification on the behaviour and performance of the component is taken into account, can then be carried out already during design of the component. Thus, the simulation strategy enables increased utilisation of the features of the casting process in the design and production of optimised and robust cast components.

\section{Conclusions}

It has been established that Digital Image Correlation can be used for characterisation and evaluation of the effects of local variations in mechanical behaviour for cast aluminium alloys. The DIC based method provides new possibilities to improve understanding of the role of local variations in microstructure on the design and performance of castings. A material characterisation model has been developed that connects SDAS and the parameters of the Hollomon equation for the current alloy. The investigation shows that:

- Local variations in microstructure cause local strain variations and possible strain redistribution during loading.

- The variation and redistribution in the strain field can be observed using DIC based methods.

- Incorporation of local material characterisation into FEM simulations enables an accurate prediction of the strain distribution in a material with local microstructural variations.

- A FEM simulation with a homogeneous material behaviour fails to predict the effects of local variations in microstructure.

- The proposed simulation strategy enables increased utilisation of the casting process in the design of optimised and robust cast components.

\section{Acknowledgements}

The Swedish Knowledge Foundation is acknowledged for financially supporting the CCSIM project connected to the CompCAST research profile at The School of Engineering, Jönköping University, within which the current work has been carried out. Volvo Group $A B$ and Ljunghäll $A B$ are greatly acknowledged for their cooperation and for providing material and components to the project. The authors would also like to thank Toni Bogdanoff for assisting in the setup of the experimental equipment.

\section{References}


[1] Stefanescu D. Modeling of Cast Iron Solidification-The Defining Moments. Metall Mater Trans A $2007 ; 38 ; 1433-47$

[2] Seifeddine S, Svensson IL. Prediction of mechanical properties of cast aluminium components at various iron contents. Mater Des 2010; 31; 6-12

[3] Seifeddine S, Wessén M, Svensson IL. Use of simulation to predict microstructure and mechanical properties in an as-cast aluminium cylinder head - comparison with experiments. Metall Sci Technol 2006; 24; 26-32

[4] Kumar P, Gaindhar JL. DAS, Solidification Time and Mechanical Properties of Al-11\%Si Alloy VProcessed Castings. AFS Trans 1997; 104; 635-8

[5] Goulart PR, Spinelli JE, Osório WR, Garcia A. Mechanical properties as a function of microstructure and solidification thermal variables of Al-Si castings. Mater Sci Eng A 2006; 421; 245-53

[6] Seifeddine S, Johansson S, Svensson IL. The influence of cooling rate and manganese content on the 6-AI5Si phase formation and mechanical properties of Al-Si based alloys. Mater Sci Eng A 2008; 490; 385-90

[7] Ceschini L, Boromei I, Morri A, Seifeddine S, Svensson IL. Microstructure, tensile and fatigue properties of the Al-10\%Si-2\% Cu alloy with different Fe and $\mathrm{Mn}$ content cast under controlled conditions. J Mater Process Technol 2009; 209; 669-79

[8] Oswalt KJ, Misra MS. Dendrite Arm Spacing (DAS): A Nondestructive Test to Evaluate Tensile Properties of Premium Quality Aluminium Alloy (Al-Si-Mg) Casting. AFS Trans 1980; 88; 845-62

[9] Cáceres CH, Davidson CJ, Griffiths JR, Wang QG. The Effect of Mg on the Microstructure and Mechanical Behavior of Al-Si-Mg casting alloys. Metall Mater Trans A 1999; 30A; 2611-8

[10] Cáceres CH, Davidson CJ, Griffiths JR. The deformation and fracture behaviour of an Al-Si-Mg casting alloy. Mater Sci Eng A 1995; 197; 171-9

[11] Wang QG. Microstructural effects on the tensile and fracture behavior of aluminum casting alloys A356/357. Metall Mater Trans A 2003; 34; 2887-99

[12] Seifeddine S, Svensson IL. The influence of Fe and $\mathrm{Mn}$ content and cooling rate on the microstructure and mechanical properties of A380-die casting alloys. Metall Sci Technol 2009; 27; 1120

[13] Sjölander E; 2011. Heat treatment of Al-Si-Cu-Mg casting alloys. Göteborg: Chalmers Reproservice

[14] Wessén M, Svensson IL, Seifeddine S, Olsson J, Schäfer W. Simulation of cooling curves, microstructures and mechanical properties in cast Al-Si based alloys. Modeling of Casting, Welding and Advanced Solidification Processes (MCWASP) XI 2006; 693-700

[15] Svensson IL, Seifeddine S, Hattel J, Kotas P, Thorborg J. On modelling of microstructure formation, local mechanical properties and stress-strain development in aluminium castings. Modeling of Casting, Welding and Advanced Solidification Processes (MCWASP) XII 2009; 129-36

[16] Olofsson J, Svensson IL. Incorporating predicted local mechanical behaviour of cast components into finite element simulations. Mater Des 2012; 34; 494-500

[17] Larsson D, Sjögren T, Svensson IL. Modelling the plastic deformation behaviour of cast irons. Giessereiforschung 2008; 60; 8-16

[18] Olofsson J, Svensson IL. The effects of local variations in mechanical behaviour - Numerical investigation of a ductile iron component. Materials \& Design 2013; 43; 264-71

[19] Cooreman S, Lecompte D, Sol H, Vantomme J, Debruyne D. Elasto-plastic material parameter identification by inverse methods: Calculation of the sensitivity matrix. Int J Solids Struct 2007; 44; 4329-41

[20] Wang Y, Lava P, Coppieters S, De Strycker M, Van Houtte P, Debruyne D. Investigation of the Uncertainty of DIC Under Heterogeneous Strain States with Numerical Tests. Strain 2012; 48; 453-62

[21] Lava P, Cooreman S, Debruyne D. Study of systematic errors in strain fields obtained via DIC using heterogeneous deformation generated by plastic FEA. Optics and Lasers in Engineering 2010; 48; 457-68 
[22] Lava P, Cooreman S, Coppieters S, De Strycker M, Debruyne D. Assessment of measuring errors in DIC using deformation fields generated by plastic FEA. Optics and Lasers in Engineering 2009; 47; 747-53

[23] Cooreman S, Lecompte D, Sol H, Vantomme J, Debruyne D. Identification of mechanical material behavior through inverse modeling and DIC. Exp Mech 2008; 48; 421-33

[24] Coppieters S, Cooreman S, Sol H, Van Houtte P, Debruyne D. Identification of the post-necking hardening behaviour of sheet metal by comparison of the internal and external work in the necking zone. J Mater Process Technol 2011; 211; 545-52

[25] Pierron F, Grédiac M. The Virtual Fields Method. New York, NY: Springer New York; 2012

[26] Sjögren T, Persson P-E, Vomacka P. Analysing The Deformation Behaviour of Compacted Graphite Cast Irons Using Digital Image Correlation Techniques. Science and Processing of Cast Iron IX 2011; 457;

[27] Fagerholt E, Dørum C, Børvik T, Laukli HI, Hopperstad OS. Experimental and numerical investigation of fracture in a cast aluminium alloy. Int J Solids Struct 2010; 47; 3352-65

[28] Ma Z, Samuel AM, Samuel FH, Doty HW, Valtierra S. A study of tensile properties in Al-Si-Cu and Al-Si-Mg alloys: Effect of B-iron intermetallics and porosity. Materials Science and Engineering: A 2008; 490; 36-51

[29] Seifeddine S, Sjögren T, Svensson IL. Variations in Microstructure and Mechanical Properties of Cast Aluminium EN AC 43100 Alloy. Metall Sci Technol 2007; 25; 12-22

[30] Gronostajski Z. The constitutive equations for FEM analysis. J Mater Process Technol 2000; 106; 40-4

[31] Hollomon JH. Tensile Deformation. Trans AIME 1945; 162; 268-90

[32] Tiryakioglu M, Staley JT, Campbell J. A comparative study of the constitutive equations to predict the work hardening characteristics of cast Al-7wt.\%Si-0.20wt.\%Mag alloys. J Mater Sci Lett 2000; 19; 2179-81

[33] Sjölander E, Seifeddine S. Influence of alloy composition, solidification rate and artificial aging on plastic deformation behaviour of Al-Si-Cu-Mg casting alloys. Int J Cast Met Res 2013; 26; 28-36

[34] MATLAB. R2012b ed: MathWorks, Inc.; 2012.

[35] MatchID. v. 4 ed. Ghent, Belgium: Catholic University College Ghent; 2013.

[36] ABAQUS Manual. Version 6.11-1 ed. Providence, USA: Dassault Systèmes Simulia Corp.; 2011.

[37] Olofsson J, Svensson IL. Casting and stress-strain simulations of a cast ductile iron component using microstructure based mechanical behavior. Modeling of Casting, Welding and Advanced Solidification Processes XIII 2012;

[38] Zolotorevsky NY, Solonin AN, Churyumov AY, Zolotorevsky VS. Study of work hardening of quenched and naturally aged Al-Mg and Al-Cu alloys. Materials Science and Engineering: A 2009; 502; $111-7$ 


\section{Figure captions}

Fig. 1: Schematic illustration of the closed chain of simulations for cast components [16]. Figure reprinted with permission from Elsevier.

Fig. 2: Schematic illustration of the experimental setup of the tensile equipment.

Fig. 3: Definition of the different gauge areas of the specimens used for analysis of the variations in strain.

Fig. 4: Microstructure obtained in the MIDDLE area of the a) SDAS10 b) SDAS25 and c) SDAS50 series.

Fig. 5: Microstructure obtained in the a) BOTTOM b) MIDDLE and c) TOP area of the SDASVAR series.

Fig. 6: Tensile curves obtained for the three different microstructural refinements. One sample per series is shown.

Fig. 7: Experimental results for a) ultimate tensile strength (UTS) and elongation b) $0.2 \%$ offset yield stress.

Fig. 8: The relationship between SDAS-value and (a) strain hardening exponent and (b) strength coefficient. The curves depict the relationship predicted by the characterisation models.

Fig. 9: Comparison between tensile curves obtained using the laser extensometer and the DIC measurements.

Fig. 10: DIC measurements of the full strain field in the SDAS10, SDAS25 and SDAS50 series at a load of about $150 \mathrm{MPa}$.

Fig. 11: Comparison between strain fields observed for the SDASVAR series using DIC and predicted by the LOCAL and HOMOGENEOUS simulations at a load of about $180 \mathrm{MPa}$.

Fig. 12: Comparison between strain fields observed for the SDASVAR series using DIC and predicted by the LOCAL and HOMOGENEOUS simulations at a load of about $300 \mathrm{MPa}$. 


\section{Figures}

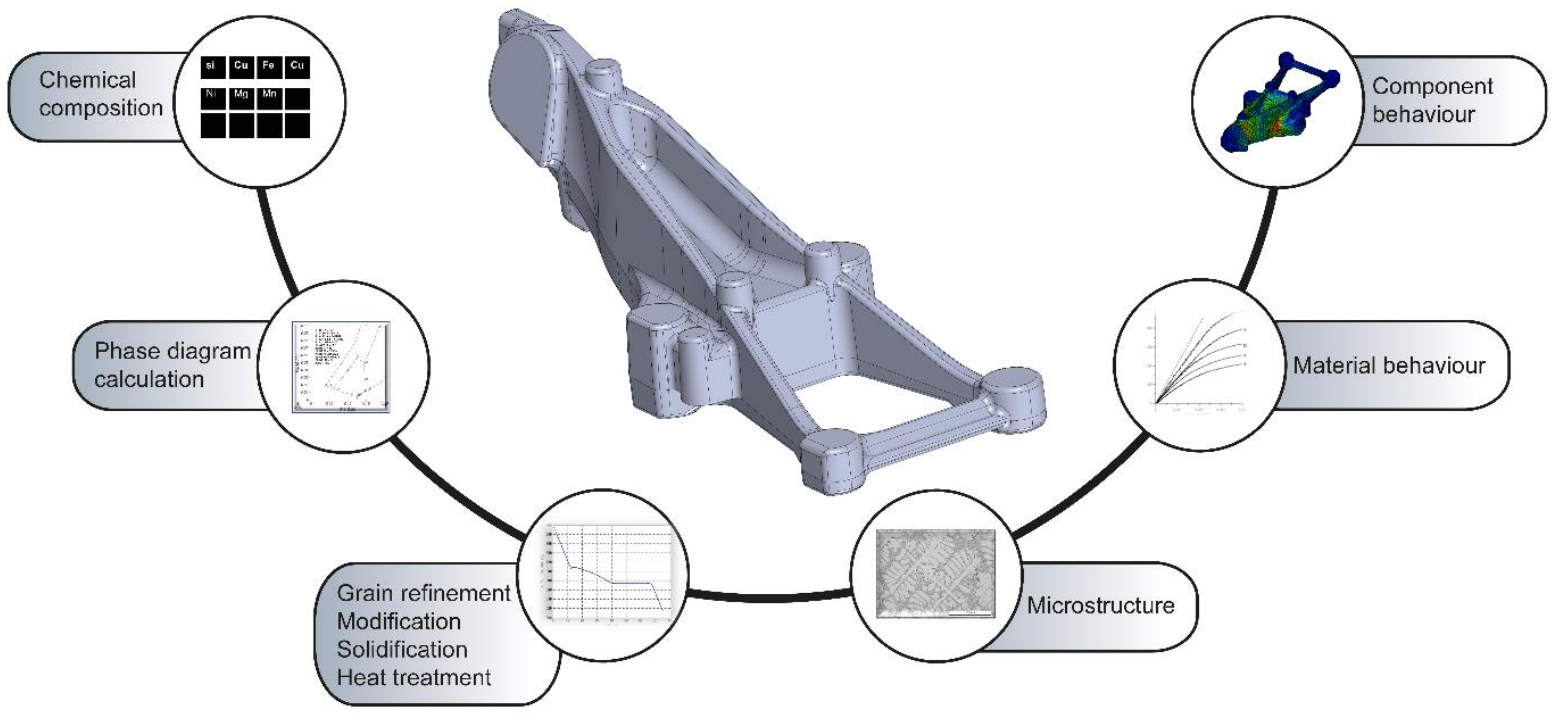

Fig. 1: Schematic illustration of the closed chain of simulations for cast components [16]. Figure reprinted with permission from Elsevier.

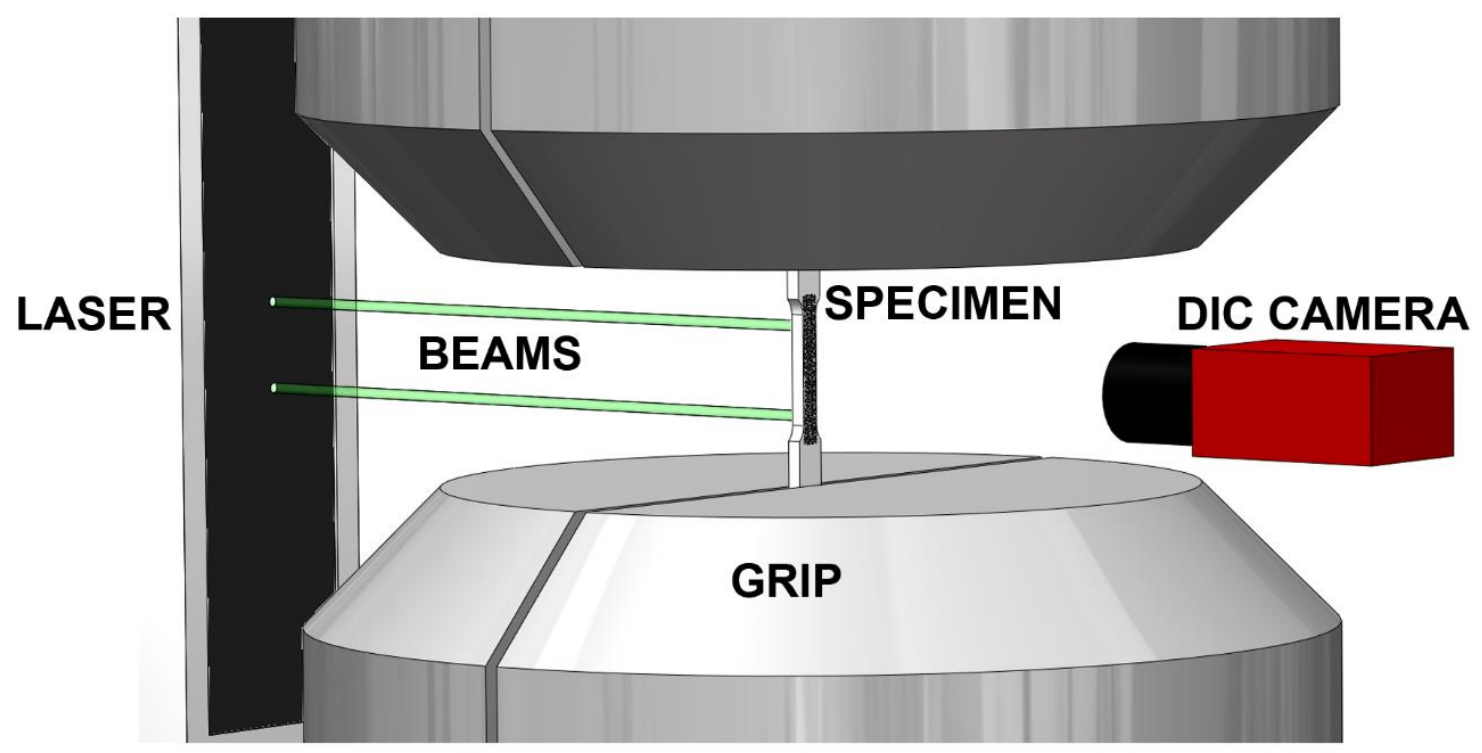

Fig. 2: Schematic illustration of the experimental setup of the tensile equipment. 


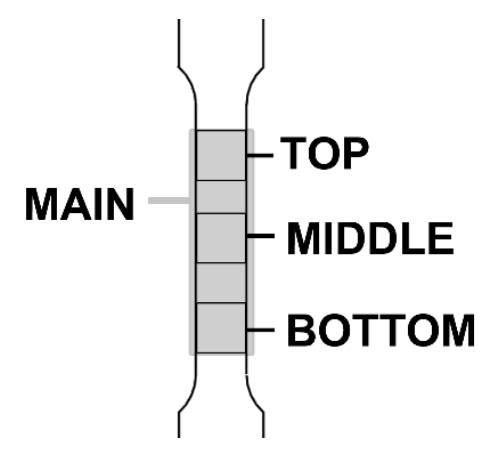

Fig. 3: Definition of the different gauge areas of the specimens used for analysis of the variations in strain.
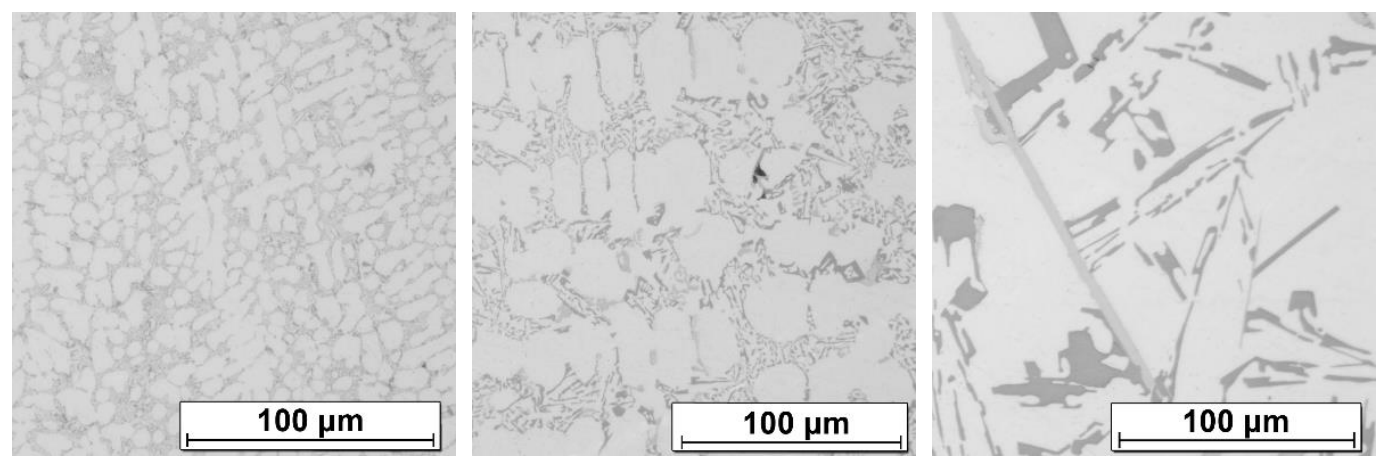

Fig. 4: Microstructure obtained in the MIDDLE area of the a) SDAS10 b) SDAS25 and c) SDAS50 series.
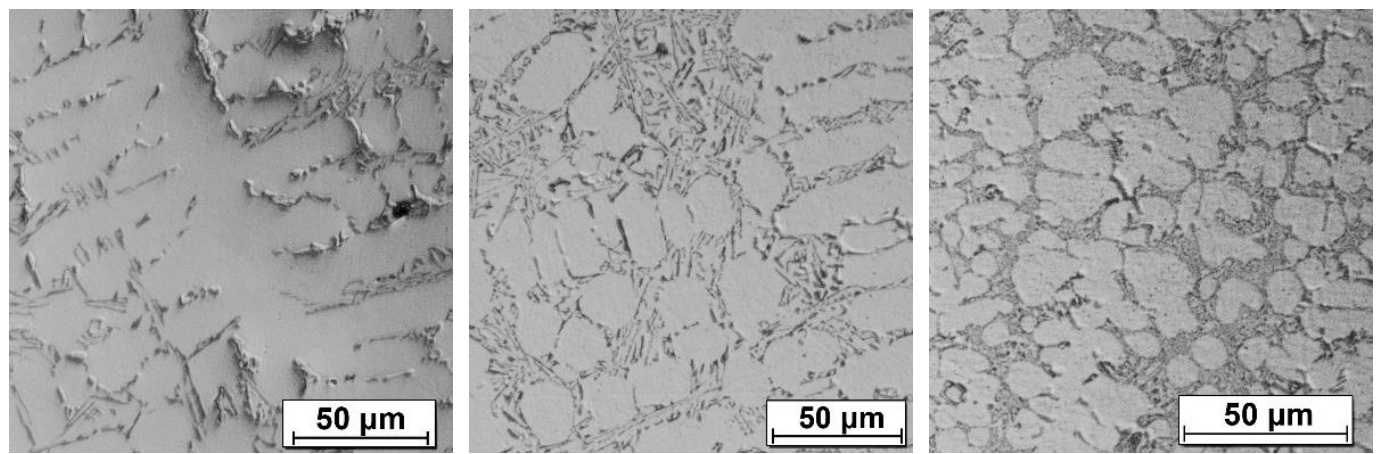

Fig. 5: Microstructure obtained in the a) BOTTOM b) MIDDLE and c) TOP area of the SDASVAR series. 


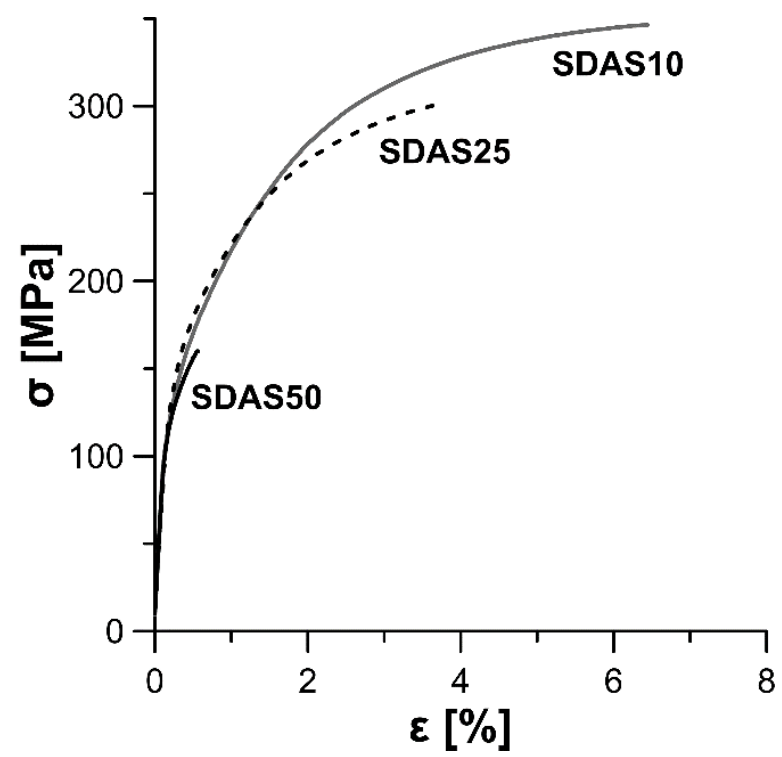

Fig. 6: Tensile curves obtained for the three different microstructural refinements. One sample per series is shown.

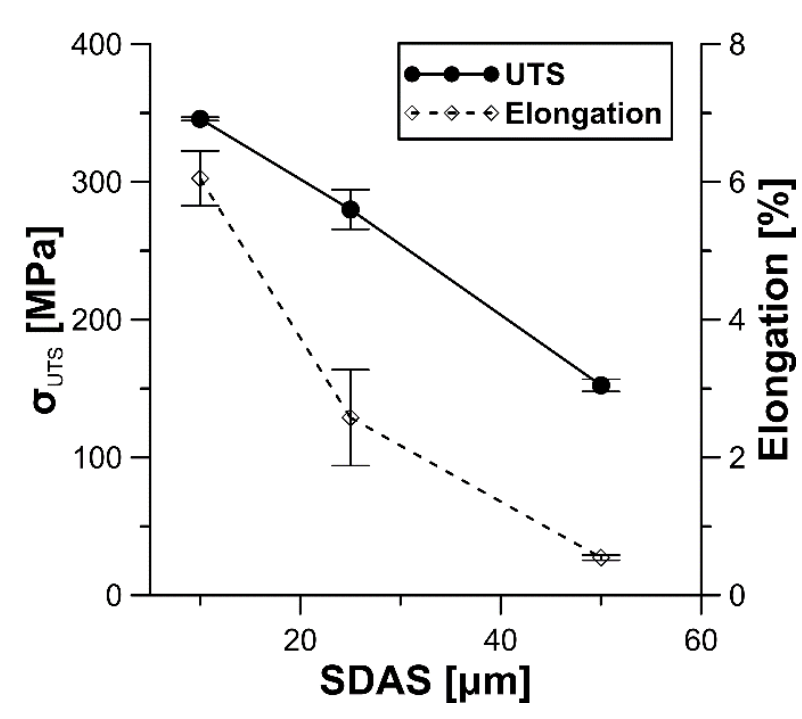

(a)

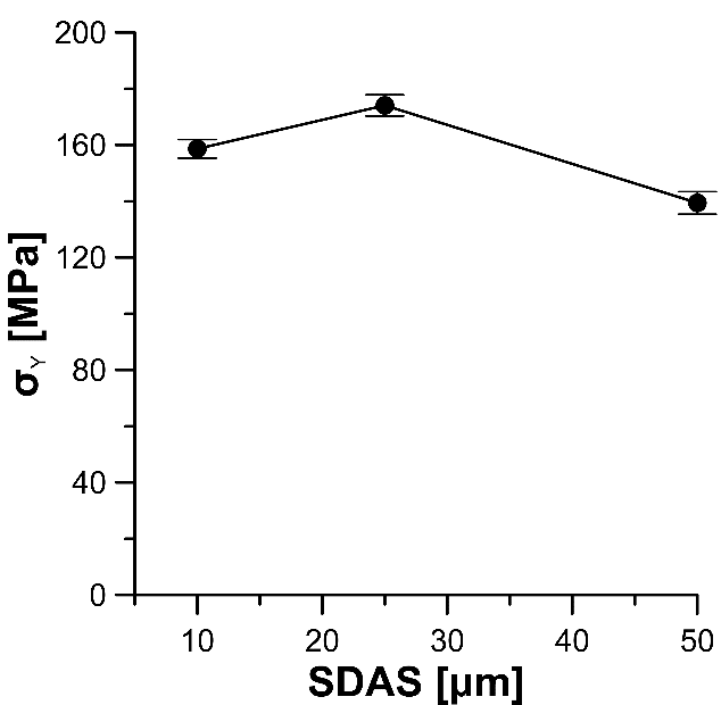

(b)

Fig. 7: Experimental results for a) ultimate tensile strength (UTS) and elongation b) $0.2 \%$ offset yield stress. 


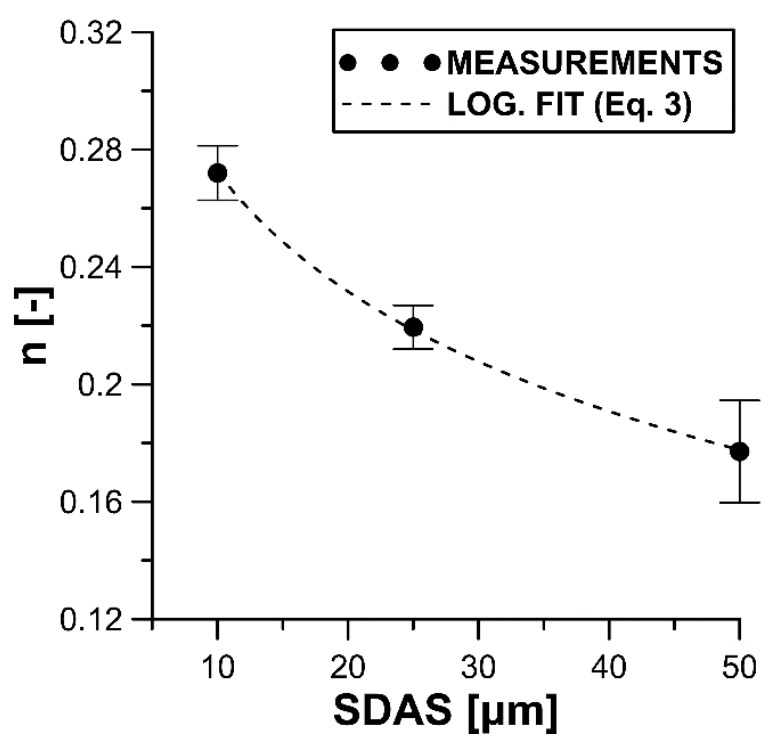

(a)

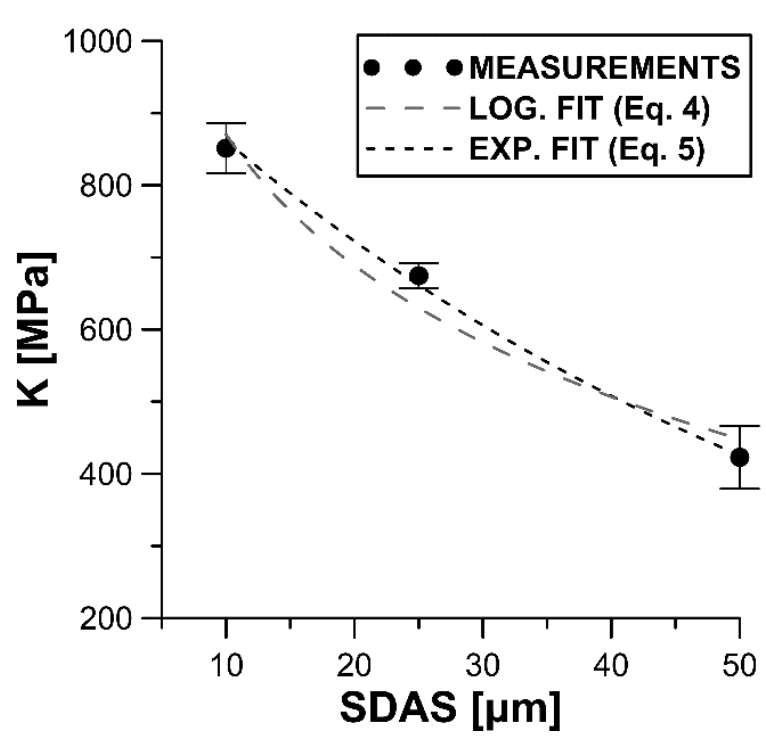

(b)

Fig. 8: The relationship between SDAS-value and (a) strain hardening exponent and (b) strength coefficient. The curves depict the relationship predicted by the characterisation models.

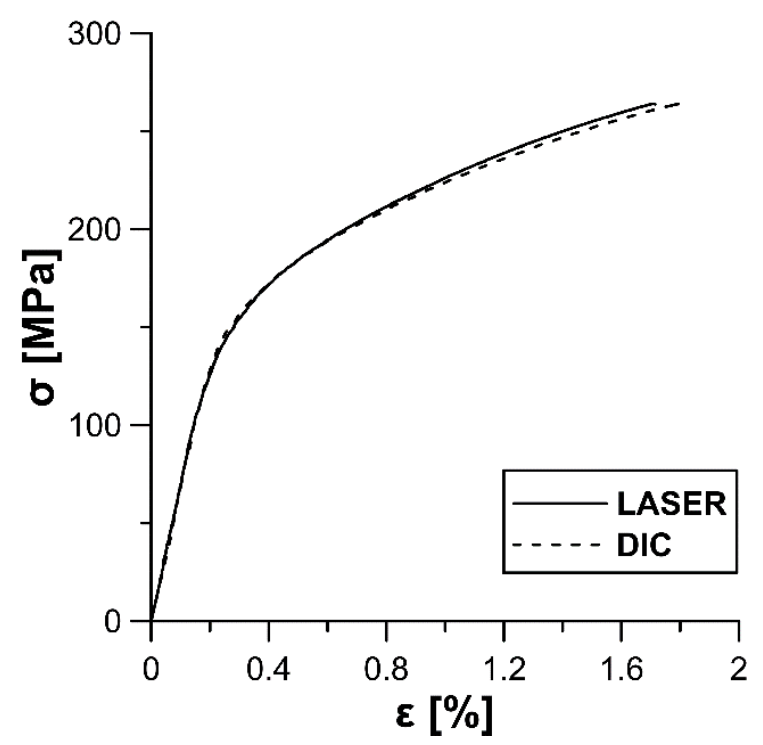

Fig. 9: Comparison between tensile curves obtained using the laser extensometer and the DIC measurements. 


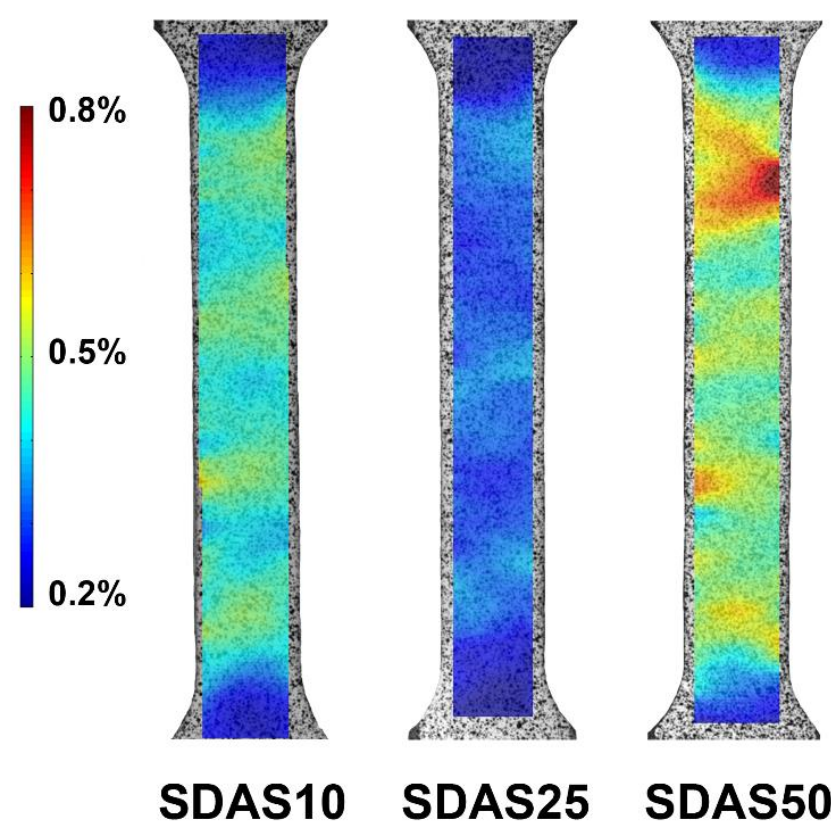

Fig. 10: DIC measurements of the full strain field in the SDAS10, SDAS25 and SDAS50 series at a load of about 150 MPa.

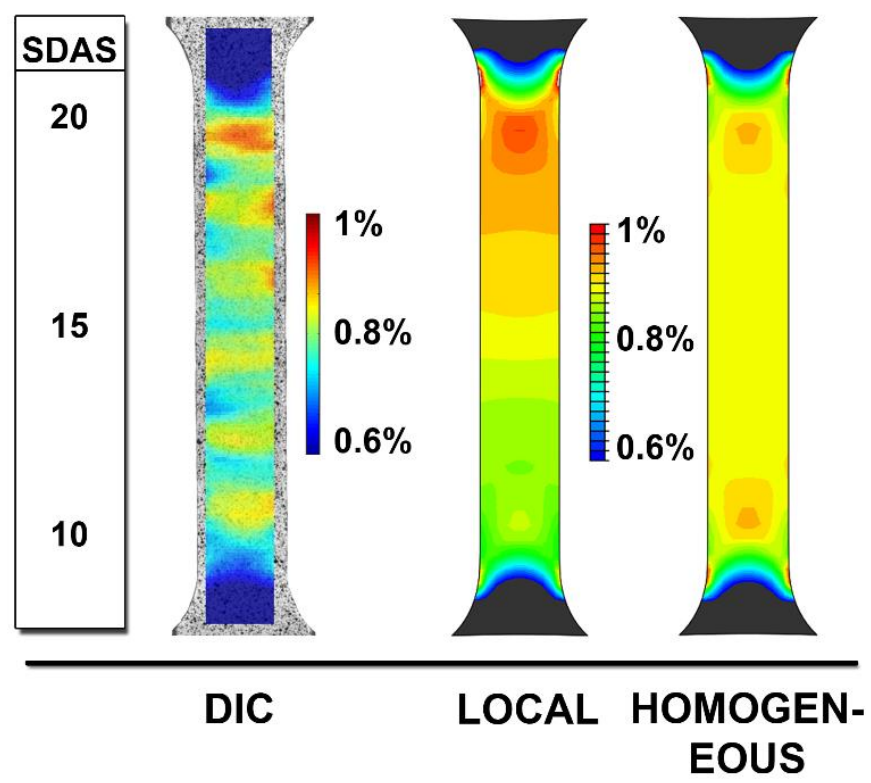

Fig. 11: Comparison between strain fields observed for the SDASVAR series using DIC and predicted by the LOCAL and HOMOGENEOUS simulations at a load of about $180 \mathrm{MPa}$. 


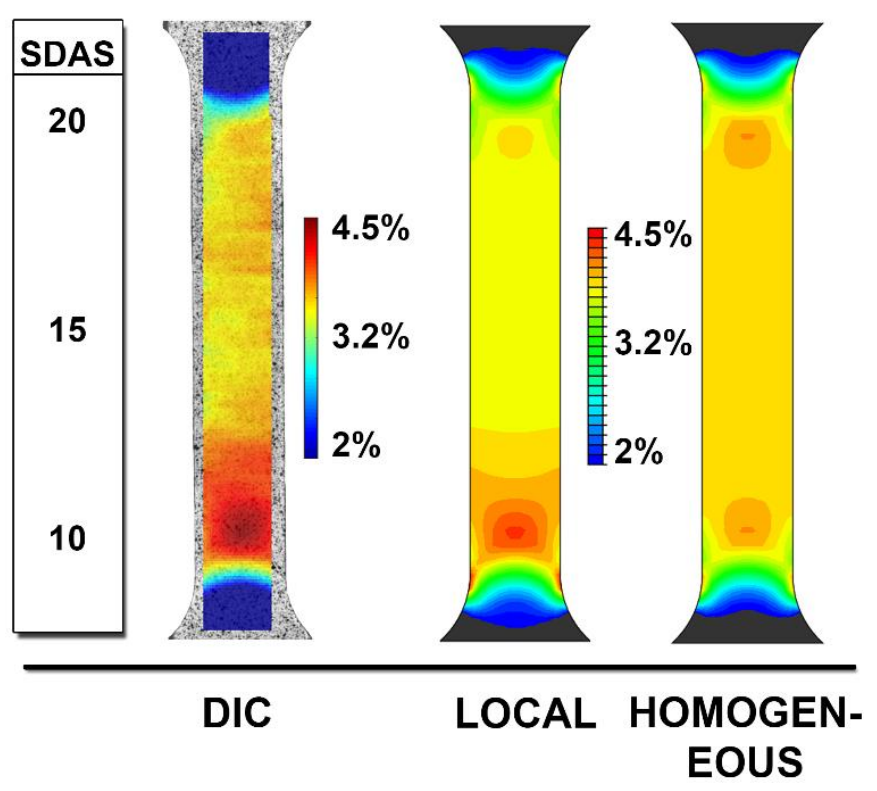

Fig. 12: Comparison between strain fields observed for the SDASVAR series using DIC and predicted by the LOCAL and HOMOGENEOUS simulations at a load of about $300 \mathrm{MPa}$. 


\section{Tables}

Table 1: Chemical composition of the investigated alloy.

\begin{tabular}{|l|l|l|l|l|l|l|}
\hline Alloying element & $\mathrm{Si}$ & $\mathrm{Cu}$ & $\mathrm{Fe}$ & $\mathrm{Mg}$ & $\mathrm{Sr}$ & Al \\
\hline Content (wt. \%) & 9.8 & 2.7 & 0.8 & 0.2 & 0.0007 & Bal. \\
\hline
\end{tabular}

Table 2: General parameters for the DIC correlations.

\begin{tabular}{|l|l|}
\hline Technique used & 2D Digital Image Correlation \\
\hline Noise & About $1 \%$ \\
\hline Subset & 15 \\
\hline Step & 5 \\
\hline Displacement spatial resolution & 15 pixels, about $0.6 \mathrm{~mm}$ \\
\hline Strain smoothing method & Polynomial - quadratic \\
\hline Spatial strain resolution & 71 pixels, about $2.8 \mathrm{~mm}$ \\
\hline Strain tensor & Logarithmic Euler-Almansi \\
\hline
\end{tabular}

Table 3: Comparison between parameters of the Hollomon equation evaluated using laser extensometer and DIC measurements. Measured standard deviations are shown within parentheses.

\begin{tabular}{|l|l|l|l|l|l|}
\hline \multicolumn{2}{|l|}{} & \multicolumn{2}{l|}{ Laser extensometer } & & \multicolumn{2}{l|}{ DIC measurements } \\
\hline Series name & $\mathrm{n}[-]$ & $\mathrm{K}[\mathrm{MPa}]$ & & $\mathrm{n}[-]$ & $\mathrm{K}[\mathrm{MPa}]$ \\
\hline SDAS10 & $0.28(0.01)$ & $879(14)$ & & $0.28(0.01)$ & $878(14)$ \\
\hline SDAS25 & $0.22(0.01)$ & $676(25)$ & & $0.22(0.01)$ & $660(22)$ \\
\hline SDAS50 & $0.18(0.02)$ & $421(44)$ & & $0.18(0.02)$ & $417(33)$ \\
\hline
\end{tabular}

Table 4: Average strain field variation according to Eq. 2 for the different series. Measured standard deviations are shown in parentheses.

\begin{tabular}{|l|l|l|l|l|}
\hline Series name & SDAS10 & SDAS25 & SDAS50 & SDASVAR \\
\hline Average Strain Variation (ASV) & $3.3(0.2)$ & $3.9(0.7)$ & $11.9(2.0)$ & $9.3(0.5)$ \\
\hline
\end{tabular}

